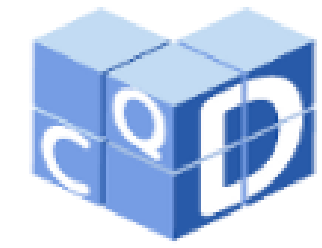

Revista Eletrônica

Paulista de Matemática

ISSN 2316-9664

Volume 10, dez. 2017

Edição Ermac

Iniciação Científica

Vinícius Aparecido Salatta

UNESPAR - Campo Mourão

vi.salatta@hotmail.com

Solange Regina dos Santos UNESPAR - Campo Mourão solaregina@gmail.com

\section{Otimização Estocástica por meio de dois problemas de programação linear com coeficientes aleatórios}

\author{
Stochastic Optmization applied for two linear programming \\ problems with random coefficients
}

\begin{abstract}
Resumo
Existem problemas de programação linear que estão comumente sujeitos a incertezas em seus dados devido a erros de medição ou previsão. Tais problemas não podem ser diretamente tratados de forma determinística, ou seja, sem levar em consideração as incertezas nos dados, pois podem gerar resultados não confiáveis, dependendo do contexto no qual se inserem. Por este motivo, destacamos nesse artigo uma das áreas da Otimização que busca levar em consideração tais incertezas na formulação dos problemas, chamada Otimização Estocástica. Nessa formulação, o modelo necessita de um conhecimento prévio de informações probabilísticas dos parâmetros de incerteza. Sendo assim, buscamos realizar um estudo de otimização sujeito a incertezas por meio da Otimização Estocástica, além de discutir a importância de se considerar as incertezas em um problema de programação linear. Para exemplificar tais discussões, utilizamos dois problemas da literatura que são resolvidos por meio da abordagem estocástica.
\end{abstract}

Palavras-chave: Otimização. Otimização Estocástica. Programação linear. Problemas sujeito à incerteza. Problema do Jornaleiro. Problema da Mistura.

\begin{abstract}
There are linear programming problems that are commonly subject to uncertainties in their data due to measurement or forecasting errors. However, such problems can not be directly treated in a deterministic way, i.e, without taking into account the uncertainties in the data, since they can generate a not very practical result depending on the context in witch it is inserted. For this reason, we highlight in this article one of the areas of optimization that seeks to take into account such uncertainties in the formulation of problems, called Stochastic Optimization. In this formulation, the model requires a prior knowledge of probabilistic information of uncertainty parameters. Thus, we seek to perform an optimization study subject to uncertainties through Stochastic Optimization, in addition to discussing the importance of considering the uncertainties in a linear programming problem. In order to exemplify such discussions, we use two literature problems that is solved through the stochastic approach.
\end{abstract}

Keywords: Optimization. Stochastic Optimization. Linear programming. Problems subject to uncertainties. Daytaler's problem. Mixture problem. 


\section{Introdução}

A busca pelo valor ótimo no contexto da programação linear surge frequentemente em problemas que envolvem uma tomada de decisão (por exemplo, uma empresa que deseja determinar a quantidade de um certo produto a ser produzido, de modo a maximizar seu lucro em um determinado mês), ao mesmo tempo em que atende toda a demanda, mão-de-obra disponível, matéria prima, etc. O modelo deste problema é caracterizado por maximizar uma função linear, sujeita a uma ou mais restrições que também são equações ou inequações lineares. Tais problemas não podem ser diretamente tratados de forma determinística, ou seja, sem levar em consideração as incertezas nos dados, pois podem gerar resultados não confiáveis, dependendo do contexto no qual se inserem. Por este motivo, destacamos duas principais abordagens que buscam trabalhar com tais problemas de otimização levando em consideração suas incertezas: a Otimização Robusta e a Otimização Estocástica. O primeiro a desenvolver um estudo acerca da Otimização Robusta foi Soyster (1973), o qual assume que os parâmetros de incerteza nesta abordagem estão variando dentro de um conjunto limitado, geralmente convexo. Já a segunda abordagem incorpora incertezas na modelagem através de variáveis aleatórias com distribuição de probabilidade conhecida (BORTOLOSSI; PAGNONCELLI, 2008).

Uma das classes importantes da Otimização Estocástica é a de modelos de recurso em dois estágios, além das abordagens espere e veja (em inglês, wait and see) e aqui e agora (em inglês, here and now). Segundo Bortolossi e Pagnoncelli (2008), o modelo com recurso em dois estágios permite que se faça uma escolha inicial (chamada de primeiro estágio) antes de se conhecer o valor dos parâmetros incertos. Após o conhecimento dos valores dos mesmos, o agente de decisão faz novas escolhas (chamadas de segundo estágio) que visam corrigir possíveis efeitos negativos gerados pela decisão de primeiro estágio. Na abordagem espere e veja, o agente de decisão pode esperar por uma realização dos coeficientes aleatórios para tomar uma decisão. Já na abordagem aqui e agora, o agente deve fazer suas escolhas antes ou sem o conhecimento das realizações dos coeficientes aleatórios. Neste último uma dificuldade adicional surge sempre que não há o conhecimento de tais coeficientes. Logo, se faz necessário especificações adicionais, uma vez que as definições habituais de otimalidade e admissibilidade não se aplicam. Diante disso, o objetivo da Otimização Estocástica é encontrar uma solução ótima dentro de todos os possíveis valores que os parâmetros aleatórios possam assumir. Isto implica que, ao invés de fixar vários cenários possíveis e resolver todos os problemas correspondentes para estes cenários, todos os cenários são incorporados em um único problema. Além desta vantagem, é possível também determinar o quanto vale a pena considerar o modelo estocástico do problema através do Valor Esperado sob Informação Perfeita (EVPI) e do Valor da Solução Estocástica (VSS). Os dois termos são denominados indicadores de qualidade, e ajudam muito na análise da estocacidade do problema.

Deste modo, o objetivo deste artigo é discutir a abordagem estocástica, bem como aplicá-la por meio de dois problemas distintos conhecidos na literatura como "o problema do jornaleiro" e "o problema da mistura", cujos detalhes podem ser encontrados em Bortolossi e Pagnoncelli (2008). No primeiro problema temos como objetivo analisar os indicadores de qualidade de modo a investigar os ganhos (ou perdas) em se considerar o modelo estocástico atribuído ao problema, ao invés de simplesmente usarmos o modelo determinístico ou aproximações determinísticas, enquanto no problema da mistura iremos aplicar as abordagens espere e veja e aqui e agora na resolução dos mesmos.

Para isto, este trabalho está dividido em nove seções. Na primeira seção apresentamos o problema do jornaleiro e formulamos seu modelo estocástico, de modo que na segunda seção resolvemos o problema utilizando alguns conceitos básicos de estatística, cálculo e álgebra 
linear. Na seção seguinte valores numéricos são atribuídos as variáveis do problema, a fim de possibilitar uma análise numérica dos indicadores de qualidade (EVPI e VSS) realizados na seção 5. Na sexta seção é apresentado o problema da mistura, de modo que as abordagens espere e veja e aqui e agora são utilizadas na resolução do problema nas seções 7 e 8 , respectivamente. Por fim, a última seção é destinada as considerações finais do presente trabalho.

Para finalizar a presente seção, lembramos que este trabalho é uma adaptação do artigo "Otimização Estocástica por meio de um problema de programação linear com coeficientes aleatórios", o qual foi apresentado e publicado no Caderno de Trabalhos Completos e Resumos, de modo que, no presente trabalho acrescentamos o problema da mistura e reestruturamos algumas das seções já existentes no artigo publicado durante o evento.

\section{0 problema do jornaleiro}

José é jornaleiro, e toda manhã vai ao editor da cidade comprar uma quantidade $x$ de jornais por um preço $c$ por unidade. Como José não possui muito dinheiro, a quantidade de jornais que José pode comprar é limitada em um intervalo de 0 a $u$. Ele vende seus jornais a um preço $q$ por unidade, além de possuir um acordo com o editor: os jornais que não forem vendidos podem ser devolvidos ao editor, o qual pagará um preço $r<c$ por unidade.

José possui uma incerteza em sua demanda diária e enfrenta um dilema: se ele comprar um número $x$ de jornais e a demanda for maior, ele perderá a oportunidade de vender mais jornais por um preço $q$. Porém, se ele comprar uma certa quantidade de jornais e a demanda for menor que esta quantidade, José terá um prejuízo em suas vendas. Portanto, vamos supor que a demanda $\omega$ é uma variável aleatória não-negativa com função densidade $f$ e função de distribuição $F$, que $y$ é o número de jornais efetivamente vendidos e que $b$ é o número de jornais possivelmente devolvidos ao editor. Sendo assim, a formulação do problema do jornaleiro é

$$
\min _{0 \leq x \leq u}\{c x+Q(x)\}
$$

em que

$$
Q(x)=\mathbb{E}_{\omega}[Q(x, \omega)]
$$

$\mathrm{e}$

$$
\begin{array}{ll}
Q(x, \omega)=\text { minimizar } & -q y(\omega)-r b(\omega) \\
\text { sujeito a } & y(\omega) \leq \omega \\
& y(\omega)+b(\omega) \leq x \\
& y(\omega), b(\omega) \geq 0
\end{array}
$$

em que $\mathbb{E}_{\omega}$ representa a esperança com respeito a demanda $\omega$.

Na equação (1), $c x$ é o custo de José ao comprar os jornais e, para a quantidade $x$ de jornais comprados, a função $-Q(x)$ representa o lucro esperado calculado sobre todos os valores possíveis de $\omega$, conforme equação (2). Sendo assim, a função $Q(x, \omega)$ pode ser calculada por meio da resolução de um problema de minimização, dado em (3). Nesse problema, a função objetivo fornece o quanto José ganha ao vender os jornais tanto para a demanda $\omega$ quanto aos jornais devolvidos ao editor a um preço $q$ e $r$, respectivamente. Note que este problema foi padronizado para um problema de minimização ao invés de maximização e, por esse motivo, invertemos os sinais da função objetivo. A primeira restrição do problema nos mostra que os 
jornais vendidos precisam ser no máximo igual a demanda, enquanto a segunda restrição é a soma dos jornais vendidos e devolvidos, os quais precisam ser no máximo igual ao total de jornais comprados.

A formulação do problema do jornaleiro se baseia em dois estágios. No primeiro estágio José precisa decidir quantos jornais irá comprar através da variável $x$. Após esta decisão, ele irá vender os jornais para uma demanda $\omega$. As variáveis de segundo estágio são a quantidade de jornais que ele vendeu $(y(\omega))$, e a quantidade de jornais devolvida ao editor $(b(\omega))$. O que José busca é determinar a quantidade exata de jornais que irá comprar de forma a maximizar o lucro esperado sob incerteza de demanda. $\mathrm{O}$ que podemos analisar incialmente é que, se José soubesse com exatidão a demanda, ele simplesmente compraria $\omega$ jornais e obteria o lucro máximo. Porém, como não é possível escolher um valor $x$ que maximize seu lucro para todos os possíveis valores de demanda $\omega$, José procura uma escolha que, em média, lhe dê o melhor lucro.

\section{Resolução do problema}

Para a resolução do problema, primeiramente é necessário determinar a solução de segundo estágio, a qual é felizmente imediata: se a demanda $\omega$ for menor do que o número de jornais comprados, então $y^{*}(\omega)=\omega$. Se for maior, então $y^{*}(\omega)=x$. Para encontrar o valor de $b^{*}(\omega)$, basta notar que a devolução de jornais ao editor só ocorre se a demanda for menor do que o número de jornais comprados.

Assim

$$
\begin{aligned}
& y^{*}(\omega)=\min \{\omega, x\} \\
& b^{*}(\omega)=\max \{x-\omega, 0\}
\end{aligned}
$$

Desta forma, podemos reescrever a formulação (2) da seguinte forma

$$
Q(x)=\mathbb{E}_{\omega}[-q \min \{\omega, x\}-r \max \{x-\omega, 0\}]
$$

A solução ótimo para o problema pode ser determinada por meio da análise do sinal da derivada primeira da função $Q(x)$, lembramos que a função analisada está limitada no intervalo de $[0, u]$.A fim de determinar a expressão para a função $Q(x)$, vamos considerar a seguinte definição.

Definição 1: Seja $g$ uma função contínua e $X$ uma variável aleatória contínua com função densidade $f$. Então

$$
\mathbb{E}[g(x)]=\int_{-\infty}^{+\infty} g(x) f(x) d x
$$

caso a integral exista.

Assim, considerando a equação (2) e a definição (1), temos que

$$
\mathbb{E}[g(t)]=\int_{-\infty}^{x} g(t) f(t) d t+\int_{x}^{+\infty} g(t) f(t) d t
$$


Caso $\omega<x$ pela equação (5) temos que $Q(x)=\mathbb{E}_{\omega}[-q \omega-r(x-\omega)]$, em que a variável $\omega$ é incerta e que $x$ é fixo. Por outro lado, caso $\omega>x$, temos que $Q(x)=\mathbb{E}_{\omega}[-q x]$. Sendo assim, substituindo estes valores na equação (6), segue que

$$
Q(x)=\mathbb{E}_{\omega}[Q(\omega, x)]=\int_{-\infty}^{x}(-q t-r(x-t)) f(t) d t+\int_{x}^{+\infty}(-q x) f(t) d t .
$$

Resolvendo esta equação obtemos

$$
Q(x)=-q x+(q-r) \int_{-\infty}^{x} F(t) d t
$$

Assim, temos que $Q^{\prime}(x)=-q+(q-r) F(x)$, de modo que as soluções possíveis são dadas a partir do teste nos estremos do intervalo e também em seu ponto crítico.

(a) Extremos do intervalo $[0, u]$

Para $x^{*}=0$ temos

$$
\begin{gathered}
c+Q^{\prime}(0)>0 \\
c+[-q+(q-r) F(0)]>0 \\
F(0)>\frac{q-c}{q-r}
\end{gathered}
$$

Para $x^{*}=u$ temos

$$
\begin{gathered}
c+Q^{\prime}(u)<0 \\
c+[-q+(q-r) F(u)]<0 \\
F(u)<\frac{q-c}{q-r}
\end{gathered}
$$

\section{(b) Ponto crítico}

Para encontrar a solução no ponto crítico, fazemos $Q^{\prime}(x)=0$. Deste modo, temos que

$$
\begin{gathered}
c+[-q+(q-r) F(x)]=0 \\
F(x)<\frac{q-c}{q-r} \\
\therefore x^{*}=F^{-1}\left(\frac{q-c}{q-r}\right)
\end{gathered}
$$

Podemos então resumir os itens (a) e (b) da seguinte forma

$$
\begin{cases}x^{*}=0, & \text { se } F(0)>\frac{q-c}{q-r} \\ x^{*}=u, & \text { se } F(u)<\frac{q-c}{q-r} \\ x^{*}=F^{-1}(x)\left(\frac{q-c}{q-r}\right), & \text { caso contrário. }\end{cases}
$$




\section{Exemplo numérico}

Suponha que o custo por jornal para o jornaleiro seja $c=10$, que o preço de venda seja $q=25$, que o preço de devolução ao editor seja $r=5$ por jornal, e que o poder de compra seja $u=150$. Além disso, considere que a demanda $\omega$ é dada por uma variável aleatória uniforme contínua definida no intervalo $[50,150]$. Então, por definição, uma variável aleatória uniforme possui função densidade de probabilidade dada por

$$
f(x \mid a, b)=\frac{1}{b-a}, a \leq x \leq b
$$

com esperança $\mathbb{E}[X]=(a+b) / 2$ e variância $\sigma^{2}(X)=(b-a)^{2} / 12$. Logo, integrando-se a densidade de $\omega$, obtemos a função de distribuição da demanda:

$$
F(\omega)=\left\{\begin{array}{cc}
\frac{\omega-50}{100}, & \text { se } 50 \leq x \leq 150 \\
1, & \text { se } x>150 \\
0, & \text { caso contrário. }
\end{array}\right.
$$

A inversa dessa função é $F^{-1}(y)=100 y+50$ no intervalo [50,150]. Usando (12), temos que a solução do problema é $x^{*}=F^{-1}(3 / 4)=125$. Assim, utilizando a formulação (1) e a equação (8), podemos dizer que o lucro esperado para este valor é

$$
\mathbb{E}_{\omega}[c x+Q(x, \omega)]=c x-q x+(q-r) \int_{50}^{x} \frac{\omega-50}{100} d \omega=-1312,5
$$

Portanto, José teria que comprar 125 jornais todos os dias para obter um lucro esperado de $R \$ 1312,50$. Esse resultado representa o valor do problema estocástico, ou RP (Recourse Problem - RP), que será apresentado a seguir.

\section{Indicadores de qualidade: EVPI e VSS}

Existem alguns conceitos referentes a Otimização Estocástica que servem de apoio para a compreensão e estudo dos resultados obtidos na seção anterior. Um deles é o Valor Esperado sob Informação Perfeita (Expected Value of Perfect information - EVPI), o qual é obtido pela diferença entre o Valor do Problema Estocástico (Recourse Problem - RP) e o valor esperado das soluções (Wait and See - WS). Assim

$$
E V P I=R P-W S
$$

Segundo Birge e Louveaux (1997), o EVPI mede o quanto o agente de decisão estaria disposto a pagar para se obter informação perfeita e precisa sobre o futuro. Sendo assim, nesta aplicação, o EVPI refere-se a quanto José estaria disposto a pagar para se obter com precisão o valor da demanda $\omega$. Para Kall e Wallace (1994), o EVPI também representa quanto se esperaria ganhar se fosse possível determinar de antemão o valor das variáveis aleatórias. Ainda, de acordo com estes autores, o EVPI é importante, porque mostra se é importante considerar a 
aleatoriedade do problema, pois não necessariamente um valor alto para o EVPI possa indicar a necessidade de se resolver o problema estocástico. Porém, quando o EVPI é baixo, temos uma indicação de que não é tão importante considerar a aleatoriedade do problema e, portanto, aproximações podem funcionar bem.

O RP representa o valor do problema estocástico, o qual foi encontrado na seção anterior. Já o WS representa o valor ótimo do problema para cada cenário, o qual neste caso está definido no intervalo de $[50,150]$.

Como o valor de RP já foi calculado, basta encontrar o valor de WS. Para isto, precisamos lembrar que o valor de $\omega$ está definido no intervalo [50,150]. Assim, como o EVPI supõe que o valor do coeficiente aleatório é conhecido, para um determinado valor $\omega$, a solução é obviamente $x^{*}=\omega$. Logo,

$$
W S=\mathbb{E}_{\omega}[c \omega-q \omega]=\mathbb{E}_{\omega}[-15 \omega]=-15 \mathbb{E}_{\omega}[\omega]=-15 \cdot \frac{50+150}{2}=-R \$ 1500,00 .
$$

O valor da esperança de $\omega$ foi calculado lembrando que $E[X]=(a+b) / 2$. Por fim, o valor do EVPI será dado por

$$
E V P I=-R \$ 1312,50+R \$ 1500,00=R \$ 187,50 .
$$

Outro importante indicador é o Valor da Solução Estocástica (Value of Stochastic Solution - VSS), o qual é obtido pela diferença entre a Solução do Valor Esperado (Expectation of the Expected Solution - EEV) e o RP, sendo dado por

$$
V S S=E E V-R P .
$$

Esse indicador represetna o quanto se ganha ao considerar o modelo estocástico. Deste modo, de acordo com Birge e Louveaux (1997), o VSS pode ser considerado como o custo de ignorar a aleatoriedade dos parâmetros na escolha de uma decisão. Neste caso, seu cálculo supõe que, em vez de resolver o problema estocástico (1) a (3), o decisor prefira resolver um problema de Valor Esperado (Expected Value - EV), que consiste em substituir todas as variáveis aleatórias do problema estocástico pelos respectivos valores esperados.

Sendo assim, temos que o VSS auxilia na comparação entre o problema estocástico e estratégias determinísticas aproximadas. Logo, quanto maior o valor do VSS, maior é o ganho em se considerar o problema estocástico em detrimento da estratégia aproximada adotada. De forma similar, se o valor for muito baixo (menor do que a definida pelo decisor), o ganho em se considerar o problema estocástico em vez do problema EV é desprezível e, portanto, podese utilizar a estratégia aproximada do valor esperado.

Para se encontrar o valor de EEV, inicialmente calcula-se a solução ótima do problema para uma demanda constante igual a média de $\omega$, a qual é obtido facilmente, uma vez que conhecendo-se o valor de $\omega$ basta comprar $x^{*}=100$ jornais para maximizar o lucro. O próximo passo então,é usar o valor de $x^{*}$ na equação (12). A solução após os cálculos será $-R \$ 1250,00$. Deste modo

$$
V S S=-R \$ 1250,00+R \$ 1312,50=R \$ 187,50 .
$$

Com base neste indicador, podemos dizer que se o jornaleiro definisse um valor para tolerância acima do resultado obtido, poderíamos dizer que o modelo estocástico não era tão importante em ser considerado em vez de outras estratégias aproximadas. 
No próximo exemplo abordaremos o problema da mistura, cuja aleatoriedade se manifesta em apenas alguns dos coeficientes.

\section{0 problema da mistura}

Como comentado na seção anterior, nesse problema a aleatoriedade se manifesta em apenas alguns dos coeficientes, neste caso, nos coeficientes das restrições de desigualdade. Para isto considere a seguinte situação: um fazendeiro consultou um engenheiro agrônomo que recomendou $7 \mathrm{~g}$ de um nutriente $\mathrm{A}$ e $4 \mathrm{~g}$ de um nutriente $\mathrm{B}$ para cada $100 \mathrm{~m}^{2}$ de terra. $\mathrm{O}$ fazendeiro dispõe de dois tipos de adubo. Cada $\mathrm{kg}$ do primeiro adubo possui $\omega_{1} \mathrm{~g}$ do nutriente A e $\omega_{2} \mathrm{~g}$ do nutriente B. Cada $\mathrm{kg}$ do segundo adubo, por sua vez, possui $1 \mathrm{~g}$ de cada nutriente. Os custos de compra de cada adubo são iguais: uma unidade monetária por kg. As quantidades $\omega_{1}$ e $\omega_{2}$ são incertas: o fabricante dos adubos garante que elas são variáveis aleatórias independentes, uniformemente distribuídas e com suportes nos intervalos $[1,4]$ e $[1 / 3,1]$, respectivamente. É preciso então, saber o quanto comprar de cada adubo para atender a necessidade de nutrientes em $100 \mathrm{~m}^{2}$ de terra, minimizando consequentemente o custo de compra. Desse modo o problema de otimização pode ser formulado da seguinte forma

$$
\begin{array}{cl}
\text { minimizar } & f\left(x_{1}, x_{2}\right)=x_{1}+x_{2} \\
\text { sujeito a } & \omega_{1} x_{1}+x_{2} \geq 7 \\
& \omega_{2} x_{1}+x_{2} \geq 4 \\
& x_{1}, x_{2} \geq 0
\end{array}
$$

em que $x_{1}$ e $x_{2}$ representam a quantidade em kg dos dois tipos de adubo disponíveis. Vale notar que o conjunto admissível desse problema depende dos valores dos coeficientes $\omega_{1}$ e $\omega_{2}$.

\section{Abordagem espere e veja}

Nessa abordagem supõe-se que o agente de decisão possa fazer a escolha dos valores de $x=\left(x_{1}, x_{2}\right)$ após a realização de $\omega=\left(\omega_{1}, \omega_{2}\right)$. As soluções ótimas assim como o valor ótimo são escolhidos em função de $\omega$. Por exemplo:

(a) Para $\omega=\left(\omega_{1}, \omega_{2}\right)=(1,1 / 3)$, temos a seguinte formulação

$$
\begin{aligned}
\text { minimizar } & f\left(x_{1}, x_{2}\right)=x_{1}+x_{2} \\
\text { sujeito a } & x_{1}+x_{2} \geq 7 \\
& 1 / 3 x_{1}+x_{2} \geq 4 \\
& x_{1}, x_{2} \geq 0
\end{aligned}
$$

Cuja solução é ilustrada pela Figura 1. 


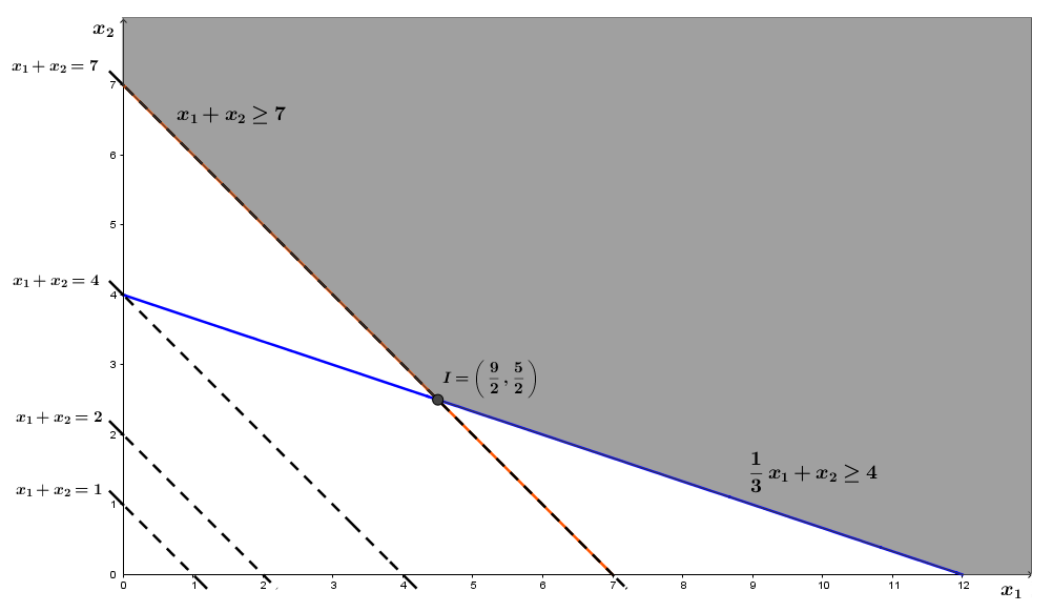

Figura 1: Conjunto admissível do problema para $\omega=(1,1 / 3)$.

Nesse caso, uma das restrições da formulação é paralela à função objetivo do problema, a qual pode ser notada pelas curvas de nível da função $x_{1}+x_{2}=k$. Esse fato acaba gerando múltiplas soluções, as quais se encontram sobre a função $x_{1}+x_{2}=7$, no intervalo $[0,9 / 2] \times[5 / 2,7]$. Para qualquer ponto dentro desse intervalo será obtido $f\left(x_{1}^{*}, x_{2}^{*}\right)=7$ como solução ótima.

(b) Para $\omega=\left(\omega_{1}, \omega_{2}\right)=(5 / 2,2 / 3)$, temos a seguinte formulação

$$
\begin{aligned}
\text { minimizar } & f\left(x_{1}, x_{2}\right)=x_{1}+x_{2} \\
\text { sujeito a } & 5 / 2 x_{1}+x_{2} \geq 7 \\
& 2 / 3 x_{1}+x_{2} \geq 4 \\
& x_{1}, x_{2} \geq 0
\end{aligned}
$$

com solução $x^{*}=(18 / 11,32 / 11)$ e valor ótimo $f\left(x^{*}\right)=4, \overline{54}$. O gráfico da solução é dado pela Figura 2.

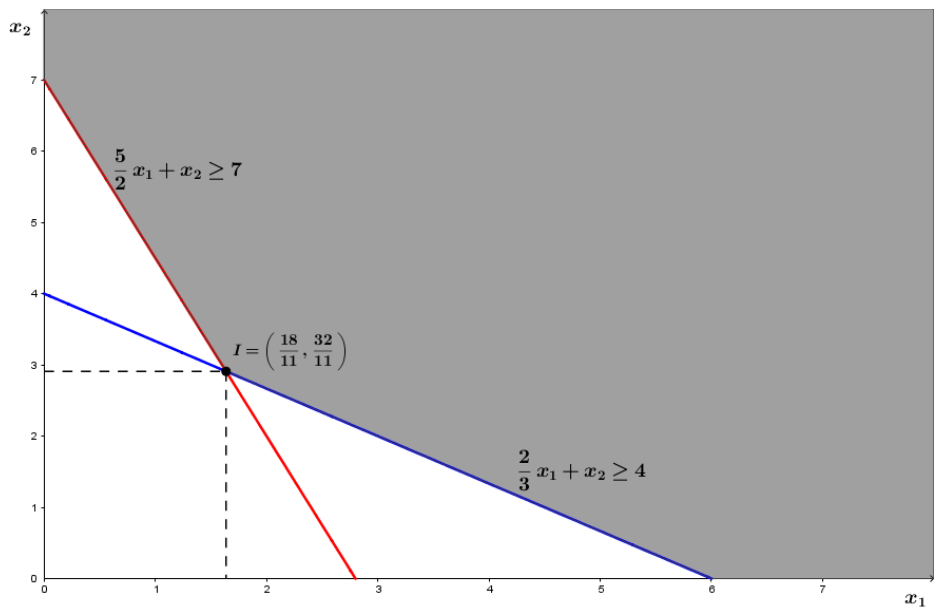

Figura 2: Conjunto admissível do problema da mistura para $\omega=(5 / 2,2 / 3)$.

(c) Para $\omega=\left(\omega_{1}, \omega_{2}\right)=(4,1)$, temos a seguinte formulação 


$$
\begin{aligned}
\text { minimizar } & f\left(x_{1}, x_{2}\right)=x_{1}+x_{2} \\
\text { sujeito a } & 4 x_{1}+x_{2} \geq 7 \\
& x_{1}+x_{2} \geq 4 \\
& x_{1}, x_{2} \geq 0
\end{aligned}
$$

Nesse item nos deparamos com uma situação semelhante ao gráfico da formulação (17) em que uma das restrições é paralela à função objetivo do problema. Deste modo podemos obter múltiplas soluções sobre a função $x_{1}+x_{2}=4$, no intervalo $[1,4] \times[0,3]$. Para qualquer ponto dentro desse intervalo obtemos a solução ótima $f\left(x_{1}^{*}, x_{2}^{*}\right)=4$.

A solução gráfica do último item pode ser observada na Figura 3.

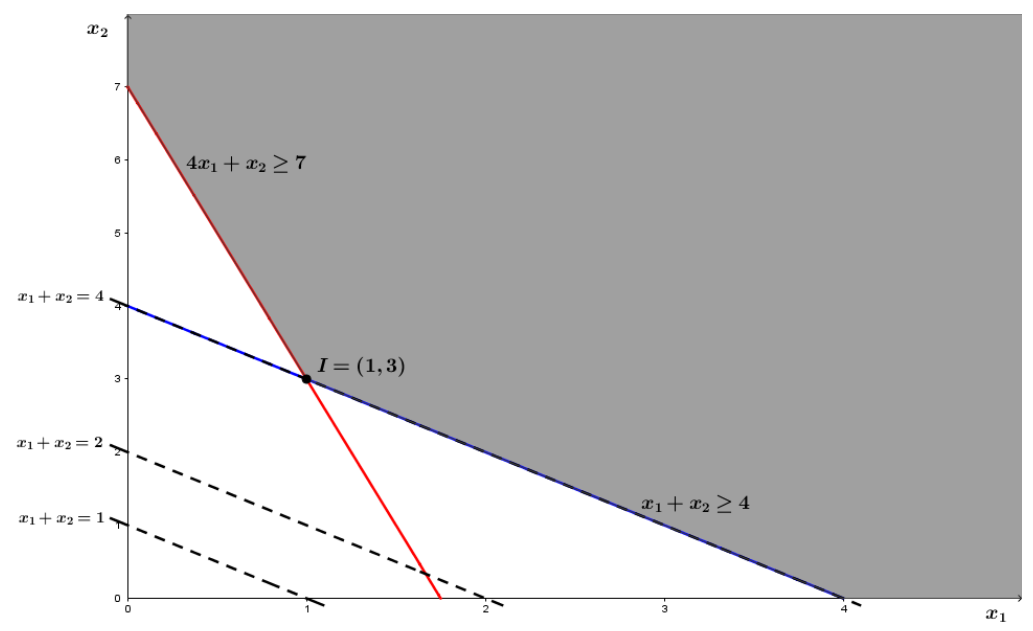

Figura 3: Conjunto admissível do problema da mistura para $\omega=(4,1)$.

Vamos mostrar agora que é possível encontrar uma solução ótima genérica para o problema (16) com $\left(\omega_{1}, \omega_{2}\right) \in \Omega=[1,4] \times[1 / 3,1]$. Para isto, queremos obter valores de $x_{1}$ e $x_{2}$ que satisfaçam ambas as restrições do problema, dadas por

$$
\left\{\begin{array}{l}
\omega_{1} x_{1}+x_{2} \geq 7 \\
\omega_{2} x_{1}+x_{2} \geq 4
\end{array}\right.
$$

Com o objetivo de eliminar o coeficiente $x_{2}$ subtraímos a primeira equação da segunda, obtendo $x_{1}\left(\omega_{1}-\omega_{2}\right) \geq 3$ e, portanto, $x_{1} \geq 3 /\left(\omega_{1}-\omega_{2}\right)$. Sabendo que $x_{1} \geq 0$ podemos concluir que $\omega_{1}>\omega_{2}$. Agora, substituindo a expressão encontrada para $x_{1}$ na segunda equação do sistema, temos

$$
\begin{aligned}
\omega_{1}\left(\frac{3}{\omega_{1}-\omega_{2}}\right)+x_{2} & \geq 7 \\
x_{2} & \geq \frac{4 \omega_{1}-7 \omega_{2}}{\omega_{1}-\omega_{2}} .
\end{aligned}
$$

Porém, sabemos que $x_{2} \geq 0$. Assim, podemos dizer que

$$
\frac{4 \omega_{1}-7 \omega_{2}}{\omega_{1}-\omega_{2}} \geq 0
$$

de modo que, após fazer as operações necessárias, temos 


$$
\frac{7}{\omega_{1}} \leq \frac{4}{\omega_{2}}
$$

Dessa forma, a partir do valor encontrado para $x_{2}$ encontramos a condição (22), a qual se satisfeita, nos fornece o par ordenado $\left(x_{1}, x_{2}\right)$ encontrado até agora. Porém, podemos encontrar um par ordenado correspondente para caso esta condição não seja satisfeita, ou seja, quando $4 / \omega_{2} \leq 7 / \omega_{1}$. A partir desta hipótese obtemos

$$
\frac{4 \omega_{1}-7 \omega_{2}}{\omega_{1}-\omega_{2}} \leq 0
$$

No entanto, sabemos que $x_{2} \geq 0$. Portanto, a única forma de satisfazer esta condição e a hipótese dada é se $x_{2}=0$, e consequentemente, $x_{1}=7 / \omega_{1}$. O valor ótimo para os dois casos é dado pela substituição de cada par ordenado $\left(x_{1}, x_{2}\right)$ na função objetivo $z=x_{1}+x_{2}$. Resumidamente, temos

$$
\left(x_{1}^{*}\left(\omega_{1}, \omega_{2}\right), x_{2}^{*}\left(\omega_{1}, \omega_{2}\right)\right)=\left\{\begin{array}{cc}
\left(\frac{3}{\omega_{1}-\omega_{2}}, \frac{4 \omega_{1}-7 \omega_{2}}{\omega_{1}-\omega_{2}}\right), & \text { se } \frac{7}{\omega_{1}} \leq \frac{4}{\omega_{2}} \\
\frac{7}{\omega_{1}}, & \text { caso contrário }
\end{array}\right.
$$

e o valor ótimo é dado pela substituição desses pontos na função objetivo do problema (16). Assim

$$
v^{*}\left(x_{1}^{*}\left(\omega_{1}, \omega_{2}\right), x_{2}^{*}\left(\omega_{1}, \omega_{2}\right)\right)=\left\{\begin{array}{cc}
\left(\frac{3}{\omega_{1}-\omega_{2}}, \frac{4 \omega_{1}-7 \omega_{2}}{\omega_{1}-\omega_{2}}\right), & \text { se } \frac{7}{\omega_{1}} \leq \frac{4}{\omega_{2}} \\
\frac{7}{\omega_{1}}, & \text { caso contrário. }
\end{array}\right.
$$

\section{Abordagem aqui e agora}

Nesta abordagem, o agente de decisão deve escolher os valores de $x$ sem conhecer os valores de $\omega$ (mas conhecendo a função de distribuição de $\omega$ ). Assim, a partir do momento que não se conhece os coeficientes, as definições habituais de otimalidade e admissibilidade não se aplicam, sendo necessárias especificações adicionais. Os tipos de especificações mais tradicionais são:

\subsection{Abolir incertezas}

O agente de decisão faz uma escolha para $\omega$ e então resolve o problema determinístico correspondente à sua escolha.

(a) Escolha "pessimista": $(\widehat{\omega}=(1,1 / 3))$. Nesse caso, o conjunto admissível é o apresentado na Figura 1 e a solução ótima correspondente é $\hat{x}=7$.

(b) Escolha "neutra": $\left(\widehat{\omega}=(5 / 2,2 / 3)=\mathbb{E}\left[\left(\omega_{1}, \omega_{2}\right)\right]\right)$. Nesse caso, o conjunto admissível é o representado na Figura 2 e a solução ótima correspondente é $\hat{x}=50 / 11=4, \overline{54}$. 
(c) Escolha "otimista": $(\widehat{\omega}=(4,1))$. Nesse caso, o conjunto admissível é o apresentado na Figura 3 e a solução ótima correspondente é $\hat{x}=4$.

A Figura 4 ilustra os casos a, b e c representados no intervalo de $\omega$.

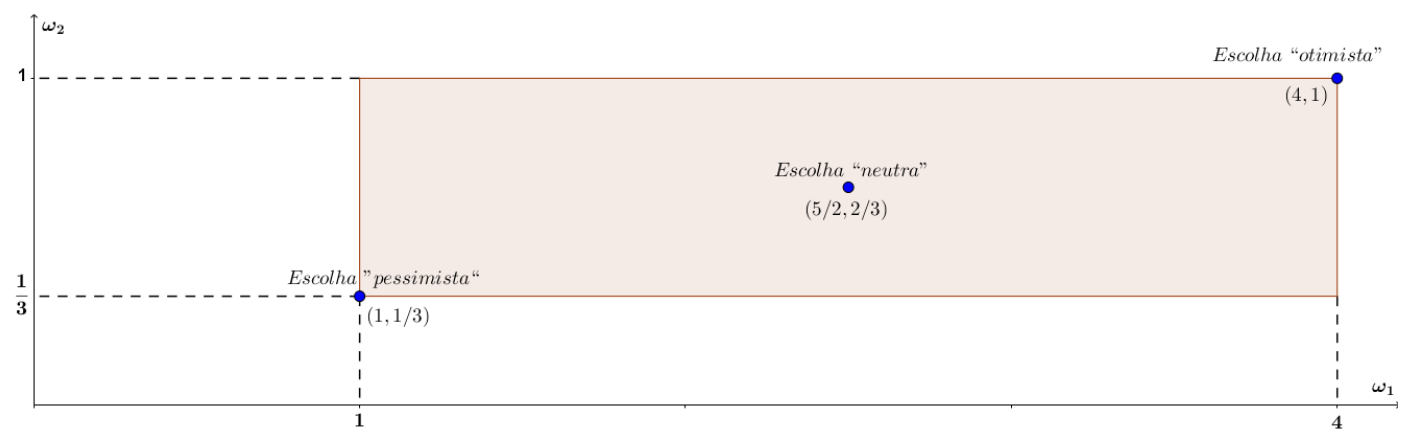

Figura 4: Intervalo de $\omega$ com os casos a, b e c.

\subsection{Incorporar riscos nas incertezas}

O agente de decisão faz uma escolha do "nível máximo de risco aceitável" e, então, ele incorpora estes elementos nas restrições do programa linear. Nesse momento, o agente decisão ainda pode escolher entre níveis de confiabilidade individuais ou conjunto. No entanto, nesse artigo vamos expor apenas o primeiro caso, uma vez que pelo nível de confiabilidade conjunto a modelagem do problema neste caso é não-linear, o qual não é de interesse desse trabalho.

\section{(a) Níveis de confiabilidade individuais}

$\mathrm{O}$ agente de decisão escolhe dois níveis de confiabilidade individuais $\alpha_{1}, \alpha_{2} \in[0,1]$ e ele decreta que $x=\left(x_{1}, x_{2}\right) \in[0,+\infty[\times[0,+\infty$ [é admissível se, e somente se

$$
\left\{\begin{array}{l}
\mathbb{P}\left(\omega_{1} x_{1}+x_{2} \geq 7\right) \geq \alpha_{1} \\
\mathbb{P}\left(\omega_{1} x_{1}+x_{2} \geq 4\right) \geq \alpha_{2}
\end{array}\right.
$$

Restrições deste tipo são denominadas restrições probabilísticas individuais (em inglês, individual chance constraints). Os riscos são definidos em termos de probabilidade de inadmissibilidade, ou seja

$$
\left\{\begin{array}{l}
\text { risco }_{1}:=\mathbb{P}\left(\omega_{1} x_{1}+x_{2} \leq 7\right) \\
\text { risco }_{2}:=\mathbb{P}\left(\omega_{1} x_{1}+x_{2} \leq 4\right)
\end{array}\right.
$$

Podemos reescrever as condições (27) de forma mais explícita, utilizando as funções distribuição $F_{1}$ e $F_{2}$ das variáveis $\omega_{1}$ e $\omega_{2}$. De fato, é possível mostrar que se $0<\alpha_{1}<1$ e $0<\alpha_{2}<1$, então

$$
\left\{\begin{array} { l } 
{ \mathbb { P } ( \omega _ { 1 } x _ { 1 } + x _ { 2 } \geq 7 ) \geq \alpha _ { 1 } } \\
{ \mathbb { P } ( \omega _ { 1 } x _ { 1 } + x _ { 2 } \geq 4 ) \geq \alpha _ { 2 } }
\end{array} \Leftrightarrow \left\{\begin{array}{l}
F_{1}^{-1}\left(1-\alpha_{1}\right) x_{1}+x_{2} \geq 7 \\
F_{2}^{-1}\left(1-\alpha_{2}\right) x_{1}+x_{2} \geq 4
\end{array}\right.\right.
$$

Primeiramente, partimos sabendo que $\mathbb{P}\left(\omega_{1} x_{1}+x_{2} \geq 7\right) \geq \alpha_{1}$. Como as variáveis $\omega$ seguem a distribuição uniforme, podemos reescrever esta probabilidade como 


$$
1-\mathbb{P}\left(\omega_{1} x_{1}+x_{2}<7\right) \geq \alpha_{1}
$$

a qual, depois de reorganizada, temos que

$$
\mathbb{P}\left(\omega_{1} x_{1}+x_{2}<7\right) \leq 1-\alpha_{1}
$$

Por definição, sabemos que $\mathbb{P}\left(\omega_{1} x_{1}+x_{2}<7\right)$ está entre 0 e 1 . Logo, $0<\alpha_{1}<1$. Agora, isolando $\omega_{1}$ na probabilidade, obtemos

$$
\mathbb{P}\left(\omega_{1}<\frac{7-x_{2}}{x_{1}}\right) \leq 1-\alpha_{1}
$$

Sabendo que $\mathbb{P}(X<x)=F(x)$, a inequação (31) ficará escrita como

$$
F\left(\frac{7-x_{2}}{x_{1}}\right) \leq 1-\alpha_{1}
$$

de modo que, após aplicar a função de distribuição inversa em ambos os lados, obtém-se

$$
\begin{gathered}
\frac{7-x_{2}}{x_{1}} \leq F_{1}^{-1}\left(1-\alpha_{1}\right) \\
F_{1}^{-1}\left(1-\alpha_{1}\right) x_{1}+x_{2} \geq 7 .
\end{gathered}
$$

A segunda equivalência da inequação (28) pode ser mostrada de forma análoga. Desta forma, o problema da mistura (16) é modelado como um problema linear da seguinte maneira

$$
\begin{aligned}
\text { minimizar } & f\left(x_{1}, x_{2}\right)=x_{1}+x_{2} \\
\text { sujeito a } & F_{1}^{-1}\left(1-\alpha_{1}\right) x_{1}+x_{2} \geq 7 \\
& F_{2}^{-1}\left(1-\alpha_{2}\right) x_{1}+x_{2} \geq 4 \\
& x_{1}, x_{2} \geq 0
\end{aligned}
$$

Dessa forma, escolhendo aleatoriamente os níveis de confiabilidade individuais $\alpha_{1}=\alpha_{2}=2 / 3$, verifica-se que

$$
F_{1}^{-1}\left(1-\alpha_{1}\right)=F_{1}^{-1}(1 / 3)=2 \text { e } F_{2}^{-1}\left(1-\alpha_{2}\right)=F_{2}^{-1}\left(\frac{1}{3}\right)=5 / 9 .
$$

Para mostrar a validade da primeira igualdade, partimos do fato de que $\omega_{1} \sim U[1,4]$. Pela definição da função distribuição inversa, podemos dizer que $F^{-1}(a)=x \Leftrightarrow F(x)=a \Leftrightarrow \mathbb{P}(X \leq x)=a$. Aplicando ao problema, $F_{1}^{-1}(1 / 3)=x \Leftrightarrow F_{1}(x)=1 / 3$ e o mesmo serve para $F_{2}^{-1}$. Assim, como $\omega$ segue a distribuição uniforme, podemos escrever que, para $F_{1}^{-1}$

$$
F_{1}\left(\omega_{1}\right)=\int_{1}^{\omega_{1}} f(t) d t=\int_{1}^{\omega_{1}} \frac{1}{3} d t
$$

que resulta em $\omega_{1}=2$. Aplicando as mesmas condições em $F_{2}^{-1}$, obtemos $\omega_{2}=5 / 9$. Desta forma o problema (34) pode ser reescrito como 


$$
\begin{aligned}
\text { minimizar } & f\left(x_{1}, x_{2}\right)=x_{1}+x_{2} \\
\text { sujeito a } & 2 x_{1}+x_{2} \geq 7 \\
& 5 / 9 x_{1}+x_{2} \geq 4 \\
& x_{1}, x_{2} \geq 0
\end{aligned}
$$

o qual, pelas equações (24) e (25) fornece a seguinte solução: $x^{*}=\left(x_{1}^{*}, x_{2}^{*}\right)=(27 / 13,37 / 13)$ com valor ótimo $v^{*}=64 / 13$.

\section{Considerações finais}

No presente artigo apresentamos dois problemas referentes a Otimização Estocástica, sendo estes o problema do jornaleiro e o problema da mistura, de modo que, o recurso em dois estágios, as abordagens espere e veja e aqui e agora possibilitaram a resolução dos mesmos. Embora tais problemas já tenham sido abordados em Bortolossi e Pagnoncelli (2008), optamos aqui por apresentar de forma detalhada os métodos empregados na resolução dos mesmos, tornando assim esta pesquisa mais atrativa para pesquisadores iniciantes em Otimização Estocástica. Para tanto, descrevemos por completo alguns conceitos referentes a Estatística e Probabilidade, além da elaboração de figuras para melhor entendimento do assunto.

No problema do jornaleiro a alternativa para sua resolução foi utilizar o recurso em dois estágios, sendo o primeiro o número de jornais comprados e o segundo como o número de jornais efetivamente vendidos e o número de jornais devolvidos. Através dos resultados obtidos, conseguimos analisar a viabilidade da aplicabilidade da abordagem estocástica no problema pelos indicadores de qualidade (EVPI e VSS), os quais facilitam a análise da solução de um problema estocástico. Destacamos que o fato da abordagem ser viável ou não no problema depende do agente decisor, o qual é responsável por definir os valores limites aceitáveis pelos indicadores. Conforme mencionado no artigo, o EVPI mede o quanto estamos dispostos a pagar para se obter informação perfeita, e o VSS informa o quanto ganhamos (ou perdemos) ao considerarmos o modelo estocástico (ou não) ao invés de se basear simplesmente em modelos determinísticos aproximados.

No problema do mistura, ao contrário do problema anterior, vemos a aleatoriedade do problema se manifestar em apenas alguns dos coeficientes. Neste caso, a abordagem escolhida para a resolução e análise do problema foram as abordagens espere e veja e aqui e agora. $\mathrm{Na}$ primeira realizamos perturbações nas variáveis aleatórias para posteriormente encontrarmos a solução do problema através de métodos clássicos para a resolução de problemas de programação linear, sendo escolhido para este artigo o método gráfico. Já na segunda abordagem nos deparamos com um cenário em que não podemos escolher ou não conhecemos os valores das variáveis aleatórias. Deste modo, os resultados foram obtidos de duas maneiras: abolindo as incertezas ou incorporando riscos de confiabilidade às mesmas.

Em virtude de cada metodologia empregada na resolução dos problemas apresentados nesse trabalho possuírem características e elementos diferenciados, acreditamos que sua comparação não é adequada. De modo que, nos limitados em apresentar detalhadamente os possíveis métodos relacionados a Otimização Estocástica voltados para o problema de pesquisa, os quais constituem o principal objetivo dessa pesquisa.

De modo geral, podemos dizer que ao considerarmos as incertezas nos problemas, em especial em problemas de programação linear, estamos mais próximos das situações que normalmente (senão sempre) acontecem no cotidiano, uma vez que estas geralmente envolvem uma tomada de decisão. Sendo assim, destacamos a relevância da abordagem estocástica neste contexto justamente por permitir mensurar tais incertezas presentes nestes problemas. 
Diante de tudo que foi mencionado, acreditamos que este artigo possa auxiliar pesquisadores da área de Agronomia, Economia, Administração, dentre outras, tendo em vista que as situações apresentadas podem ser aplicadas a problemas reais os quais, em geral, possuem incerteza em sua formulação.

\section{Referências}

BIRGE, J. R.; LOUVEAUX, F. Introduction to stochastic programming. New York: Springer Science \& Business Media, 1997.

BORTOLOSSI, H. J.; PAGNONCELLI, B. K. Uma introdução à otimização sob incerteza. In: SIMPÓSIO DE PESQUISA OPERACIONAL E LOGÍSTICA DA MARINHA, 11., 2008, Rio de Janeiro. [Trabalhos apresentados.] Rio de Janeiro, 2008. Disponível em:

<http://bernardokp.uai.cl/otimizacao-sob-incerteza.pdf>. Acesso em: 27 nov. 2017.

KALL, P.; WALLACE, S. W. Stochastic programming. New York: Springer, 1994.

SOYSTER, A. L. Convex programming with set-inclusive constraints and applications to inexact linear programming. Operations Research, v.21, p.1154-1157, 1973. 